\title{
$c w r A$, a gene that specifically responds to cell wall damage in Staphylococcus aureus
}

\author{
Correspondence \\ Jianshi Tao \\ jianshi.tao@novartis.com
}

Received 3 November 2009

Revised 18 January 2010

Accepted 12 February 2010

\author{
Carl J. Balibar, Xiaoyu Shen, Dorothy McGuire, Donghui Yu, \\ David McKenney and Jianshi Tao
}

\author{
Department of Infectious Diseases, Novartis Institutes for BioMedical Research, 500 Technology \\ Square, Cambridge, MA 02139, USA
}

\section{INTRODUCTION}

Staphylococcus aureus is a facultatively aerobic Grampositive bacterium that naturally inhabits the skin and mucous membranes of mammals. It is notorious for causing both nosocomial and community-acquired infections, resulting in a variety of human diseases ranging from mild wound infections to more serious ailments that include septic shock, endocarditis and osteomyelitis (Archer, 1998). Multidrug-resistant S. aureus have emerged rapidly in the clinic, and cases of community-acquired infections are on the rise worldwide (Klevens et al., 2006), necessitating the need for novel drug discovery efforts.

Given its essential role in supporting cell viability and interacting with the external environment during infection (Amako et al., 1982; Giesbrecht et al., 1998; Navarre \& Schneewind, 1999), the staphylococcal cell wall has proven to be a desirable and successful target for antibacterial intervention. In the past decade, transcriptomic studies have become increasingly utilized in the drug discovery

Abbreviations: CCCP, carbonyl cyanide m-chlorophenylhydrazone; RLU, relative light units; TEM, transmission electron microscopy; UPP, undecaprenyl pyrophosphate.

A supplementary figure, showing a time course of lysis in response to lysostaphin, and two supplementary tables, showing the luminescence signal of RN4220/ux1 in response to various antibiotics, and the luminescence signal of RN4220/ux2 in response to various antibiotics, are available with the online version of this paper. process, especially in elucidating the mechanism of action of novel chemical agents. Previous transcriptional profiling studies have attempted to define the staphylococcal response to treatment with cell wall-targeting antibiotics. When the transcriptomes of several strains of $S$. aureus treated with oxacillin, D-cycloserine, bacitracin or vancomycin were combined, a set of 15 upregulated and two downregulated genes were found to be common to all four studies (Kuroda et al., 2003; McAleese et al., 2006; McCallum et al., 2006; Utaida et al., 2003). These upregulated genes, fmt, htrA, prsA, sgtB, vraS, murZ, tcaA, SA02100, SA02101, SA02112, SA02583, SA02723, SA02724, SA02811 and SA02872, and downregulated genes, atl and SA00427, have been proposed to encompass a coordinately regulated group termed the 'cell wall stimulon'.

One gene from this stimulon, SA2343 (N315) or SAOUHSC_02872 (NCTC 8325), has incited particular interest. In genetic studies that we performed on S. aureus, SA2343 was shown to be upregulated 100-500 fold when any of the genes in the mevalonate pathway, responsible for biosynthesis of the isoprenoid precursor isopentenyl pyrophosphate (IPP), is downregulated (Balibar et al., 2009). Given that IPP is the building block for the cell wall carrier lipid undecaprenyl pyrophosphate (UPP), which is essential in peptidoglycan biosynthesis (Wilding et al., 2000), this massive overexpression is presumed to be a response to cell wall damage. This upregulation of SA2343 was more than 20 -fold greater than the upregulation of any 
other gene in the cell wall stimulon. Further stimulating interest in SA2343 is the fact that in a separate study it was shown to be the highest-fold upregulated gene in several clinical vancomycin intermediate-resistant $S$. aureus (VISA) strains (McAleese et al., 2006).

Herein, we seek to understand the role that gene SA2343, which we have renamed cwrA for cell wall responsive for antibiotics, plays in S. aureus. Using genetic and phenotypic readouts we seek to define the conditions under which this gene is expressed and the role that it plays in the physiology of $S$. aureus.

\section{METHODS}

Constructing luciferase integration vectors. For cloning purposes, S. aureus integration vector pCL84 (Lee et al., 1991) was first double-digested with PstI/SalI restriction enzymes, blunt-ended with T4 DNA polymerase, and religated with T4 DNA ligase to generate pCL84 $\triangle$ Pst $\triangle$ Sal. The luxABCDE gene cluster was amplified from $S$. aureus strain Xen29 (Xenogen) with the primers 5'-CCAGGATCCTGGTCGACAAAAGGAGGATACGTATGAAATTTGGAAACTTTTTGCTTACAT-3' and ' 5'-ATTCCATGGAATTCTTAACTATCAAACGCTTCGGT-3' (restriction sites underlined), and cloned into the BamHI and EcoRI sites in pCL84 $\Delta$ Pst $\Delta$ Sal. This resulted in pCL84/Lux, which contained an optimal ribosome-binding site (RBS) for $\operatorname{luxA}$, and BamHI and SalI sites upstream of the luxA gene for cloning of desired promoters to generate transcriptional fusions. Either the putative promoter of $c w r A$ (from positions -228 to $-145^{\prime}$ to the $c w r A$ ORF), using the primers $5^{\prime}$-GGCGGATCCAACTCCTAATAACTTATTTACTTGT- ${ }^{\prime}$ and $5^{\prime}$-AAAGTCGACTTTATATTTCTCTTACTCACATTG- $3^{\prime}$, or the putative promoter plus the $c w r A$ ORF, using the primers 5'-GGCGGATCCAACTCCTAATAACTTATTTACTTGT-3' and 5'-TAAGTCGACTTAAAAGAAATCAGATGGGTTA-3', was amplified and then cloned into the BamHI/SalI sites in pCL84/Lux, generating pCL84/PcwrA-Lux or pCL84/ PcwrA_ORFLux, respectively.

In order to generate the translational fusion vector, the region between the third codon of $c w r A$ and the start of luxA in pCL84/ PcwrA_ORFLux was deleted. Utilizing a unique SnaBI site adjacent to the start codon of $\operatorname{lu} x A$, the primers $5^{\prime}$-AATCAATTACGTATGA-

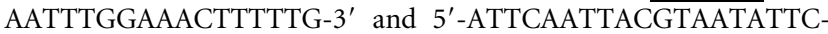
TCATCGCTATCATAC- $3^{\prime}$ were used to amplify the entire plasmid outside the deleted region. The linear PCR product was then digested with SnaBI and ligated to yield pCL84/PcwrA_4aaLux. All plasmids were propagated in Escherichia coli TOP10 cells (Invitrogen) and sequenced (Agencourt Bioscience) to verify that the constructs were correct.

\footnotetext{
Integration of reporter constructs into the S. aureus chromosome. The integration plasmids pCL84/PcwrA-Lux, pCL84/ PcwrA_ORFLux and pCL84/PcwrA_4aaLux were separately transformed into $S$. aureus strain CYL316, which is RN4220 carrying a plasmid-borne phage integrase gene that facilitates the site-specific integration of pCL84-based plasmids into the attB locus on the $S$. aureus chromosome (Lee et al., 1991). Successful integrants were selected through tetracycline resistance conferred by the tet ${ }^{R}$ cassette on pCL84, and subsequently verified by PCR screening. The corresponding clones were named RN4220lux1, RN4220lux2 and RN4220lux3, respectively.
}

Testing of compounds on reporter constructs. In a 96-well format, overnight cultures of the lux reporter strains were diluted 50fold in fresh Mueller-Hinton Broth (MHB; Becton Dickinson), and
$99 \mu \mathrm{l}$ of the diluted cells was then aliquoted to each well of a 96-well flat-bottomed white polystyrene tissue-culture plate (Costar). One microlitre of the compound to be tested, at various concentrations, was then added to each well. The cells were grown at $37{ }^{\circ} \mathrm{C}$ with shaking at 225 r.p.m. The luciferase signal in each well was determined using a PHERAstar microplate reader (BMG LABTECH) at various time points. Erythromycin, chloramphenicol, rifampicin, novobiocin, carbonyl cyanide $m$-chlorophenylhydrazone (CCCP), oxacillin and bacitracin were purchased from Sigma. Triclosan, vancomycin, penicillin, imipenem and ciprofloxacin were purchased from USP. Daptomycin was purchased from Cubist Pharmaceuticals and hymeglusin was obtained from the Novartis Natural Products Unit (NPU).

For stress responses, hydrochloric acid $(0.04-90 \mathrm{mM})$, sodium hydroxide $(0.04-90 \mathrm{mM})$ or hydrogen peroxide $(0.003-5.9 \%$, v/v) was added to cultures, which were grown and measured for luminescence as described above. Heat and cold shock were performed by growing cultures at 43 and $6{ }^{\circ} \mathrm{C}$, respectively.

Construction of cwrA deletion strain. In order to delete $c w r A$, $500 \mathrm{bp}$ upstream and $500 \mathrm{bp}$ downstream of the gene were PCRamplified and cloned into either side of an erythromycin-resistance cassette that was inserted into the multiple cloning site of pBAD-Kan (Guzman et al., 1995) (ATCC). The upstream region was amplified using the primers $5^{\prime}$-AAGAATTCTTGTAATCTGTCCACCATCC-3' and $5^{\prime}$-AGCGGTACCGCTATCATACTCCTTTATAT-3', digested, and cloned between the EcoRI and KpnI sites. The downstream region was amplified using the primers $5^{\prime}$-ACGGATCCGTCACCTAAGAATTGCAAATCC- $3^{\prime}$ and $5^{\prime}$-TTAGTCGACTAACACTTTTTCAATACGGC- $3^{\prime}$, digested, and cloned between the BamHI and SalI sites. The resulting plasmid was transformed into RN4220, and successful integrants were selected by screening for resistance to erythromycin. Double-crossover deletions were then selected by restreaking single-crossover integrants and PCR screening for the replacement of $c w r A$ by the erythromycin cassette, with concomitant loss of the plasmid backbone. The cwrA deletion was then transduced using phage $80 \alpha$ into SH1000 to yield SH1000 $\Delta$ cwrA : : erm.

RNA isolation and transcriptional profiling. Overnight cultures of

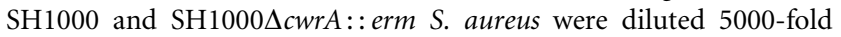
into fresh MHB. Cultures were grown with vigorous shaking at $37{ }^{\circ} \mathrm{C}$ to $\mathrm{OD}_{600} 0.5$, at which point $30 \mathrm{ml}$ was harvested by centrifugation. The remaining culture was grown for an additional $12 \mathrm{~h}$ until the culture reached saturation, at which point an equivalent of $15 \mathrm{ml}$ of culture at $\mathrm{OD}_{600} 1.0$ was harvested by centrifugation. Cells were resuspended in $4 \mathrm{ml} \mathrm{H}_{2} \mathrm{O}$ and $8 \mathrm{ml}$ RNAProtect Bacteria reagent (Qiagen), incubated for at least $30 \mathrm{~min}$, pelleted by centrifugation, and then frozen at $-80{ }^{\circ} \mathrm{C}$ until further use. RNA was extracted from cell pellets by using a Purescript RNA isolation kit (Gentra Systems) with an initial treatment with $0.3 \mu \mathrm{g}$ lysostaphin $\mu^{-1}$ (Sigma) to achieve cell lysis. A total of $100 \mu \mathrm{g}$ total RNA was treated with $7 \mathrm{U}$ RNase-free DNase I (Qiagen) and then further purified by using an RNeasy Plus Mini Kit (Qiagen). RNA quality was interrogated by reacting with formaldehyde loading dye running on a $1 \%$ agarose denaturing gel with MOPS gel running buffer from a NorthernMax kit (Applied Biosystems). Final RNA samples were quantified by using a NanoDrop spectrophotomoter.

Gene expression profiling was performed using Affymetrix GeneChips designed to interrogate over $3300 \mathrm{~S}$. aureus ORFs and both the forward and the reverse orientation of more than 4800 intergenic regions. Fifteen micrograms of RNA was reverse-transcribed, and the cDNA was fragmented, labelled and hybridized to the GeneChip $S$. aureus genome array using the standard prokaryotic GeneChip protocol supplied by Affymetrix. GeneChip data were obtained by scanning with an Affymetrix autoloading scanner, and data were normalized and compared by using GeneSpring 7.2 analysis software 
(Agilent Technologies). All experiments were done in triplicate using independent RNA samples.

Transmission electron microscopy (TEM) imaging. Overnight cultures of SH1000 and SH1000 $\mathrm{cwrA}$ : : erm were grown in MHB, harvested at stationary phase, and analysed by TEM at the W. M. Keck Microscopy Facility at the Whitehead Institute, Cambridge, MA, USA. Cells were fixed in $2.5 \%$ glutaraldehyde $(\mathrm{w} / \mathrm{v}), 3 \%$ paraformaldehyde $(\mathrm{w} / \mathrm{v})$ and $5 \%$ sucrose $(\mathrm{w} / \mathrm{v})$ in $0.1 \mathrm{M}$ sodium cacodylate buffer, $\mathrm{pH} 7.4$, pelleted, and post-fixed in $1 \% \mathrm{OsO}_{4}(\mathrm{w} / \mathrm{v})$ in veronal-acetate buffer. The cell pellet was stained in block overnight with $0.5 \%$ uranyl acetate (w/v) in veronal-acetate buffer, $\mathrm{pH}$ 6.0, then dehydrated and embedded in Spurr's resin. Sections were cut on a Reichert Ultracut E microtome with a Diatome diamond knife at a thickness setting of $50 \mathrm{~nm}$, and stained with uranyl acetate and lead citrate. The sections were examined using an FEI Tecnai Spirit transmission electron microscope at $80 \mathrm{kV}$ and photographed with an AMT charge-coupled device (CCD) camera.

In vivo autolysis assays. Overnight cultures of $\mathrm{SH} 1000$ and SH1000 $\Delta$ cwrA:: erm were diluted $1: 100$ into $\mathrm{MHB}+1 \mathrm{M} \mathrm{NaCl}$ with or without $350 \mathrm{ng}$ phosphomycin $\mathrm{ml}^{-1}$ and grown at $37^{\circ} \mathrm{C}$ for $6 \mathrm{~h}$. Cells were then pelleted, washed with an equal volume of cold water, and resuspended in $50 \mathrm{mM}$ Tris, $\mathrm{pH} 8$, and $1 \%$ Triton X-100 (v/v). Cultures were then incubated at $37{ }^{\circ} \mathrm{C}$ with shaking at 225 r.p.m. and $\mathrm{OD}_{600}$ readings were taken every $20 \mathrm{~min}$ using a SpectraMax Plus 384 plate reader.

Preparation of autolytic extracts. Overnight cultures of SH1000 and SH1000 $\Delta c w r A:$ erm were diluted $1: 100$ into $11 \mathrm{MHB}+1 \mathrm{M}$ $\mathrm{NaCl}$ and grown at $37^{\circ} \mathrm{C}$ for $5 \mathrm{~h}$. Cells were then pelleted and washed with $500 \mathrm{ml}$ cold water, and cell pellets were frozen at $-80{ }^{\circ} \mathrm{C}$ until further use. Three types of autolytic extract were prepared: freezethaw, LiCl and SDS. For freeze-thaw extracts, cell pellets were resuspended in $4 \mathrm{ml} 50 \mathrm{mM}$ potassium phosphate buffer, $\mathrm{pH}$ 7.3, and subjected to three rounds of freezing at $-80{ }^{\circ} \mathrm{C}$ for $1 \mathrm{~h}$ and thawing at $37{ }^{\circ} \mathrm{C}$ for $10 \mathrm{~min}$. Cells were pelleted by centrifugation at $8000 \mathrm{~g}$, and the supernatant was concentrated to $\sim 250 \mu \mathrm{l}$ in an Amicon Ultra-4 $10 \mathrm{kDa}$ molecular weight cut off (MWCO) centrifugal filter unit (Millipore). For LiCl extracts, cell pellets were resuspended in $4 \mathrm{ml} 3 \mathrm{M} \mathrm{LiCl}$ and extracted for $1 \mathrm{~h} \mathrm{at} 4{ }^{\circ} \mathrm{C}$. Cells were pelleted by centrifugation at $8000 \mathrm{~g}$, and the supernatant was buffer-exchanged 35000 -fold in an Amicon Ultra-15 $10 \mathrm{kDa}$ MWCO centrifugal filter unit (Millipore) into $500 \mu \mathrm{l} 50 \mathrm{mM}$ Tris, $\mathrm{pH}$ 7.5. For SDS extracts, cell pellets were resuspended in $1 \mathrm{ml} 4 \%$ SDS and extracted for $1 \mathrm{~h}$ at room temperature.

Crude cell wall preparation. Bacterial cell pellets remaining from autolytic extracts were boiled in $10 \mathrm{ml} 8 \%$ SDS for $1 \mathrm{~h}$, washed five times with $50 \mathrm{ml} \mathrm{H}_{2} \mathrm{O}$ until all traces of SDS were removed, incubated in $5 \mathrm{ml} 30 \mathrm{mM}$ Tris, $\mathrm{pH} \mathrm{8.0,} \mathrm{with} 200 \mu \mathrm{g} \mathrm{ml}^{-1}$ proteinase $\mathrm{K}$ (Qiagen) at $50{ }^{\circ} \mathrm{C}$ for $1.5 \mathrm{~h}$, boiled in $8 \%$ SDS again for $1 \mathrm{~h}$, and then washed five times with $50 \mathrm{ml} \mathrm{H}_{2} \mathrm{O}$ until all traces of SDS were removed. The remaining pellet was lyophilized to dryness.

In vitro autolysis assay. Crude cell walls were resuspended in $50 \mathrm{mM}$ potassium phosphate buffer, $\mathrm{pH} 7.3$, to an initial $\mathrm{OD}_{600}$ of 0.6 , and incubated with $15 \mu \mathrm{g} \mathrm{ml}^{-1}$ of either freeze-thaw or $\mathrm{LiCl}$ autolytic extract at $37^{\circ} \mathrm{C}$ in a total volume of $100 \mu$ l. Readings were taken in a 96-well plate using a SpectraMax Plus 384 plate reader.

Zymogen profiles. Crude cell walls were incorporated into a polyacrylamide resolving gel ( $10 \%$ acrylamide/ $0.26 \%$ bisacrylamide) at $0.2 \%(\mathrm{w} / \mathrm{v})$. Ten micrograms of total protein from SDS- and LiClextracted supernatants was electrophoresed for $1 \mathrm{~h}$ at $170 \mathrm{~V}$. The gel was then washed in $11 \mathrm{H}_{2} \mathrm{O}$ for $1 \mathrm{~h}$ and incubated overnight in 11 $50 \mathrm{mM}$ Tris, pH 7.5, $5 \mathrm{mM} \mathrm{MgCl}_{2}$ and $0.1 \%(\mathrm{v} / \mathrm{v})$ Triton X-100 at
$37{ }^{\circ} \mathrm{C}$. Peptidoglycan hydrolases were visible as clearings in the opaque gel that could be imaged against a dark background.

MIC and lysostaphin susceptibility testing. For MIC testing, $S$. aureus $\mathrm{SH} 1000$ or $\mathrm{SH} 1000 \Delta c w r A$ : : erm was inoculated into MHB and allowed to grow for $4 \mathrm{~h}$. A $0.5 \mathrm{ml}$ volume of culture was transferred into $2 \mathrm{ml}$ of sterile saline in order to achieve the turbidity of a 0.5 McFarland standard. Subsequently, $0.5 \mathrm{ml}$ of the 0.5 McFarland dilution was transferred into $4.5 \mathrm{ml}$ MHB. A $1.5 \mu \mathrm{l}$ volume of this culture and $1 \mu \mathrm{l}$ of the compound under test at various concentrations were inoculated into $100 \mu \mathrm{l} \mathrm{MHB}$ in a 96-well plate, grown at $37^{\circ} \mathrm{C}$, and scored for growth the following day.

For lysostaphin susceptibility, overnight cultures of S. aureus SH1000 or SH1000 $\Delta c w r A:$ erm grown in MHB were diluted fivefold into $20 \mathrm{mM}$ Tris, $\mathrm{pH}$ 8.0, 2 mM EDTA buffer. Lysostaphin was added at a concentration of $1.7 \mu \mathrm{g} \mathrm{ml}^{-1}$ and lysis was monitored continuously at $600 \mathrm{~nm}$ while shaking at $37{ }^{\circ} \mathrm{C}$ in a SpectraMax Plus 384 plate reader.

In vivo sepsis model. A lethal sepsis model was performed by infecting female CD-1 mice (20-25 g, Charles River Laboratories) with varying infectious doses of SH1000 and SH1000 $\Delta c w r A:: \mathrm{erm}$, ranging from $3.5 \times 10^{6}$ to $4.2 \times 10^{8}$ c.f.u. per mouse. Studies described in this report were approved by the Institutional Animal Care and Use Committee (IACUC) of the Novartis Institutes for BioMedical Research Inc. To prepare the inocula, $25 \mathrm{ml} \mathrm{MHB}$ was inoculated with $20 \mu \mathrm{l} \mathrm{S}$. aureus overnight culture and incubated for $16 \mathrm{~h}$ at $36{ }^{\circ} \mathrm{C}$ while shaking at 150 r.p.m. These cultures were then diluted $1: 5$, $1: 25$ and $1: 125$ in sterile saline. The original culture and three dilutions were further diluted $1: 1$ in $10 \%$ porcine stomach mucin (Sigma), and $0.3 \mathrm{ml}$ was used for infection by intraperitoneal injection. Tenfold serial dilutions of the inocula were plated for c.f.u. determination. Following infection, mice were monitored for 5 days, and the $\mathrm{LD}_{50}$ for both strains was calculated by probit analysis.

\section{RESULTS}

\section{Generation and evaluation of lux reporter strains}

In order to determine the conditions under which cwrA is expressed we generated two transcriptional fusion constructs in which the bacterial $\operatorname{lu} x A B C D E$ cassette (Kadurugamuwa et al., 2003) was appended downstream of either the putative promoter for cwrA (RN4220lux1) or cwrA itself (RN4220lux2) (Fig. 1a). In both constructs, translation of the lux cassette is initiated from a strong RBS independent from the RBS for cwrA. A single copy was integrated into the genome of RN4220, and these reporter strains were subsequently monitored for their responses to various applied stresses.

We treated the lux reporter strains with a variety of antibiotics that possess different mechanisms of action, including the peptidoglycan pentapeptide binder vancomycin, the $\beta$-lactam penicillin-binding protein (PBP) inhibitors oxacillin, penicillin $G$ and imipenem, the UPP binder bacitracin, the enoyl pyruvate transferase (MurA) inhibitor phosphomycin, the HMG-CoA synthase (MvaS) inhibitor hymeglusin, the DNA gyrase inhibitors ciprofloxacin and novobiocin, the $50 \mathrm{~S}$ ribosome inhibitors chloramphenicol and erythromycin, the enoyl-acyl carrier protein reductase (FabI) inhibitor triclosan, the lipopeptide daptomycin, the RNA polymerase inhibitor rifampicin, 
(a) RN4220lux 1

Promoter aaatataaagtcgacaaaaggaggatacgtatg $\operatorname{lu} x A$

RN4220lux2

RBS Start Stop RBS Start

Promoter aaatataaggagtatgatagcgatg $c w r A$ taagtcgacaaaaggaggatacgtatg luxA

RN4220lux3

RBS Start

Promoter aaatataaaggagtatgatagcgatgagaatattacgt lux $A$

(b)

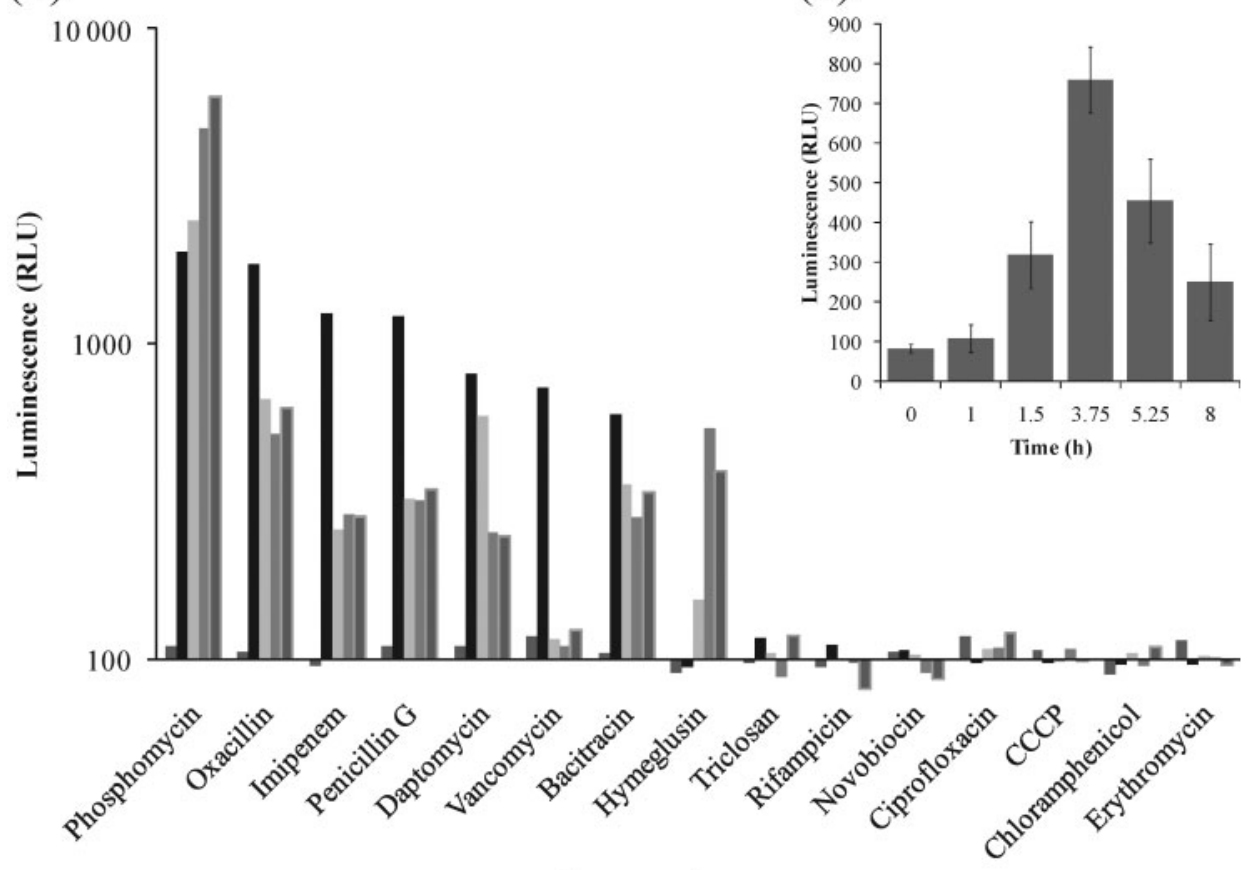

Compound treatment

Fig. 1. Reporter strains and results of monitoring $c w r A$ expression in response to antibiotic treatment. (a) DNA sequence of the two transcriptional and one translational construct in which luxABCDE is fused to cwrA. (b) Luminescence signal in RLU generated by RN4220/ux2 in response to treatment with various antibiotics at $3 \mu \mathrm{g} \mathrm{ml}^{-1}$. The first dark-grey, second black, third light-grey, fourth mid-grey and fifth dark-grey bars represent $0,1.5,2.5,3.5$ and 6 h post antibiotic addition, respectively. (c) Luminescence signal in RLU generated by RN4220/ux3 in response to treatment with $3 \mu \mathrm{g}$ phosphomycin ml $\mathrm{m}^{-1}$ at various time point post antibiotic addition. Error bars, SD $(n=3)$.

and the ionophore CCCP. Fig. 1(b) shows a summary of the luciferase signals obtained from the RN4220lux2 strain under these various treatment conditions. Although both the RN4220lux1 and the RN4220lux2 strain yielded essentially identical results at the full range of antibiotic concentrations from several fold above to several fold below MIC (Supplementary Tables S1 and S2), for simplicity Fig. 1(b) shows the results from the RN4220lux2 strain at $3 \mu \mathrm{g} \mathrm{ml}^{-1}$ antibiotic treatment only. The basal luciferase expression level was below the detection limit, showing background readings of about 100 relative light units (RLU). However, when RN4220lux2 was treated with compounds that block any aspect of cell wall biosynthesis, the resulting cultures produced luminescence readings significantly above background, without exception. In contrast, all other antibiotics with non-cell wall inhibition mechanisms, including DNA replication, RNA transcription, protein translation, fatty acid biosynthesis and membrane disruption, failed to elicit any detectable luminescence. It is interesting to note that 
daptomycin, for which the mechanism of action has not been clearly defined, also triggered a significant cell wall inhibition-like response. A recent transcription profiling study indicates that daptomycin does induce the cell wall stimulon (Muthaiyan et al., 2008), consistent with our findings, indicating that there is probably a cell wall component to the mechanism of action of daptomycin.

In addition to antibiotic treatment, we subjected the reporter strains to various environmental stresses including heat, cold, alkaline, acid and oxidative shock. None of these conditions was capable of inducing a luminescence signal above background (data not shown). This demonstrates that the specific and robust response of cwrA to cell walltargeting agents is not due to a secondary response that can be directly produced by certain environmental stresses.

cwrA is a hypothetical protein of 63 amino acids containing an $\mathrm{N}$-terminal membrane-spanning domain and an extracellular C-terminal domain, based on TOPCONS membrane protein topology prediction (Bernsel et al., 2009). In order to determine whether the predicted protein was actually translated, a third lux fusion strain was generated, RN4220lux3, in which the first four amino acids of cwrA were fused to the $\mathrm{N}$ terminus of $\operatorname{lu} x A$ (Fig. 1a). In this construct, the translation of the luciferase cassette is dependent on the endogenous RBS of $c w r A$. As can be seen in Fig. 1(c), this reporter did indeed respond robustly to phosphomycin treatment, verifying that $c w r A$ is not only transcribed but also translated in response to cell walltargeting agents. Finally, to investigate whether cwrA is autoregulatory, a $c w r A$ knockout was generated in the RN4220lux1 background (RN4220lux1 $\Delta c w r A::$ erm) and retested for its response to cell wall-targeting antibiotics. There was no appreciable difference between the two strains in the luciferase signal generated due to cell wall biosynthesis inhibition (data not shown), indicating that cwrA is not autoregulatory.

\section{Generation of cwrA knockouts and transcriptional profiling}

After identification of the conditions under which $c w r A$ is induced, we sought to understand the biological role that cwrA plays in S. aureus. To this end, a knockout strain was initially constructed in RN4220 in which cwrA was deleted and replaced with the erythromycin cassette. This knockout was then transduced into an SH1000 background, in which $\sigma^{\mathrm{B}}$-dependent signalling has been restored (Horsburgh et al.,

Table 1. Genes downregulated in $\mathrm{SH} 1000$ upon deletion of $c w r A$ during exponential growth

\begin{tabular}{|c|c|c|c|}
\hline $\begin{array}{l}\text { SH1000 ORF } \\
\text { (SAOUHSC no.) }\end{array}$ & Gene & Description & $\begin{array}{c}\text { Fold change } \\
\text { ( } \Delta c w r A / \text { wild-type })\end{array}$ \\
\hline 01603 & & Conserved hypothetical protein & 0.34 \\
\hline 01297 & & Conserved hypothetical protein & 0.48 \\
\hline 00175 & $m s m X$ & Multiple sugar-binding transport ATP-binding protein, putative & 0.33 \\
\hline 00176 & & Bacterial extracellular solute-binding protein, putative & 0.40 \\
\hline 00356 & & Conserved hypothetical protein & 0.27 \\
\hline 00437 & treP & PTS enzyme II, phosphoenolpyruvate-dependent, trehalose-specific & 0.06 \\
\hline 00438 & $\operatorname{treC}$ & $\alpha$-Glucosidase & 0.08 \\
\hline 00439 & treR & Trehalose operon transcriptional regulator & 0.11 \\
\hline 00690 & & Conserved hypothetical protein & 0.49 \\
\hline 00830 & & Conserved hypothetical protein & 0.49 \\
\hline 00838 & & Conserved hypothetical protein & 0.41 \\
\hline 01601 & malA & $\alpha$-D-1,4-Glucosidase & 0.47 \\
\hline 01602 & malR & Maltose operon transcriptional repressor & 0.41 \\
\hline 02270 & $n r g A$ & Ammonium transporter & 0.41 \\
\hline 02662 & scrA & PTS system, sucrose-specific IIBC component & 0.28 \\
\hline 02759 & & Conserved hypothetical protein & 0.47 \\
\hline 02827 & & Conserved hypothetical protein & 0.40 \\
\hline 02831 & & Haloacid dehalogenase-like hydrolase & 0.34 \\
\hline 02836 & & Conserved hypothetical protein & 0.47 \\
\hline 02853 & & Conserved hypothetical protein & 0.39 \\
\hline 02872 & $c w r A$ & Conserved hypothetical protein & 0.03 \\
\hline 02975 & & PTS system, fructose-specific IIABC component, putative & 0.16 \\
\hline 02976 & $\operatorname{man} A$ & Mannose-6-phosphate isomerase, class I & 0.21 \\
\hline 03028 & & Conserved hypothetical protein & 0.36 \\
\hline 03046 & & Helix-turn-helix domain protein & 0.26 \\
\hline 00838 & & Conserved hypothetical protein & 0.46 \\
\hline 00919 & & Conserved hypothetical protein & 0.49 \\
\hline 01729 & & Conserved hypothetical protein & 0.50 \\
\hline
\end{tabular}


2002), yielding the strain SH1000 $\Delta c w r A$ :: erm. The knockout strain did not appear to have any delay in in vitro growth kinetics. Furthermore, co-culturing experiments, in which equal dilutions of both the wild-type and knockout strain were inoculated into the same flask and then plated after the culture grew overnight to saturation, yielded equal numbers of c.f.u., indicating no defect in growth (data not shown). The only phenotype that we could initially observe was a clumping of the knockout strain when the culture reached high saturation.

With little in vitro growth difference, transcriptional profiling was performed in order to ascertain whether there were any significant differences in gene expression between the wild-type and knockout strains. Transcriptional profiling was performed on cells in both exponential and stationary growth phases. The deletion of $c w r A$ caused a downregulation of 28 genes (Table 1 ) and upregulation of 32 genes (Table 2) in exponential phase, and a downregulation of five genes and an upregulation of 12 genes (Table 3) in stationary phase. These alterations in transcription represent a threshold of at least twofold, and have a $P$ value of $\leqslant 0.05$ as determined by one-way ANOVA. Consistent with the lack of observable difference in growth phenotype, knocking out cwrA had little effect on the transcriptome of $S$. aureus.

The greatest number of transcriptional changes were observed during exponential growth. The genes most prominently downregulated were for the transport and metabolism of sugars, including trehalose, maltose, sucrose and fructose. Contrarily, the entire lac operon, responsible for the uptake and catabolism of lactose, was upregulated in response to $c w r A$ deletion. In addition to carbohydrate metabolic genes, there were several cell envelope-associated determinants that showed altered expression. A putative phosphate (Pho) regulon was upregulated in the mutant strain. Although not studied in S. aureus, phosphate transport systems have been shown to be important for

Table 2. Genes upregulated in $\mathrm{SH} 1000$ upon deletion of $c w r A$ during exponential growth

\begin{tabular}{|c|c|c|c|}
\hline $\begin{array}{l}\text { SH1000 ORF } \\
\text { (SAOUHSC no.) }\end{array}$ & Gene & Description & $\begin{array}{c}\text { Fold change } \\
(\Delta c w r A / \text { wild-type })\end{array}$ \\
\hline 01783 & & Conserved hypothetical protein & 2.05 \\
\hline 01388 & & Conserved hypothetical protein & 3.27 \\
\hline 00035 & & Conserved hypothetical protein & 2.53 \\
\hline 00206 & lct $E$ & L-Lactate dehydrogenase & 2.46 \\
\hline 00248 & lytM & Peptidoglycan hydrolase & 2.04 \\
\hline 00868 & & Conserved hypothetical protein & 2.19 \\
\hline 00868 & & Conserved hypothetical protein & 2.20 \\
\hline 00869 & $d l t A$ & D-Alanine-D-alanyl carrier protein ligase (Dcl) & 2.40 \\
\hline 00870 & $d l t B$ & Secretion of D-alanyl Dcp & 2.25 \\
\hline 00871 & $d l t C$ & D-Alanyl carrier protein (Dcp) & 2.36 \\
\hline 00872 & $d l t D$ & Selection of Dcp for ligation with D-alanine & 2.24 \\
\hline 01331 & & Conserved hypothetical protein & 2.27 \\
\hline 01384 & phoU & Phosphate uptake regulatory protein, putative & 2.10 \\
\hline 01385 & pstB & Phosphate $\mathrm{ABC}$ transporter ATP-binding protein, putative & 2.22 \\
\hline 01386 & pstA & Phosphate $\mathrm{ABC}$ transporter, permease protein, putative & 2.11 \\
\hline 01387 & pstC & Phosphate $\mathrm{ABC}$ transporter, transmembrane subunit, putative & 2.40 \\
\hline 01389 & pstS & Phosphate $\mathrm{ABC}$ transporter, periplasmic phosphate-binding protein, putative & 2.11 \\
\hline 01737 & aspS & Aspartyl-tRNA synthetase & 2.41 \\
\hline 01738 & hisS & Histidyl-tRNA synthetase & 2.22 \\
\hline 02261 & $\operatorname{agr} B$ & Accessory gene regulator protein $B$ & 2.44 \\
\hline 02333 & $s c e D$ & Lytic transglycosylase & 2.19 \\
\hline 02449 & lacG & 6-Phospho- $\beta$-galactosidase & 3.45 \\
\hline 02450 & lacE & PTS system, lactose-specific IIBC component & 3.19 \\
\hline 02451 & $\operatorname{lacF}$ & PTS system, lactose-specific IIA component & 3.39 \\
\hline 02452 & lacD & Tagatose-1,6-diphosphate aldolase & 3.46 \\
\hline 02453 & lacC & Tagatose-6-phosphate kinase & 3.12 \\
\hline 02454 & $\operatorname{lacB}$ & Galactose-6-phosphate isomerase & 2.99 \\
\hline 02455 & lacA & Galactose-6-phosphate isomerase & 2.82 \\
\hline 02571 & $s s a A$ & Secretory antigen precursor & 2.47 \\
\hline 02932 & bet $A$ & Choline dehydrogenase & 2.35 \\
\hline 02937 & cudT & Choline transporter & 3.09 \\
\hline 03033 & & High-affinity nickel transporter, putative & 2.16 \\
\hline
\end{tabular}


Table 3. Transcriptional changes in $\mathrm{SH} 1000$ upon deletion of $c w r A$ during stationary growth

\begin{tabular}{|c|c|c|c|}
\hline $\begin{array}{l}\text { SH1000 ORF } \\
\text { (SAOUHSC no.) }\end{array}$ & Gene & Description & $\begin{array}{c}\text { Fold change } \\
(\Delta c w r A / \text { wild-type })\end{array}$ \\
\hline \multicolumn{4}{|l|}{ Downregulated } \\
\hline 01554 & & PV83 orf 27-like protein & 0.43 \\
\hline 02021 & & phi ETA orf 63-like protein & 0.47 \\
\hline 02057 & & dUTP pyrophosphatase & 0.37 \\
\hline 02872 & cwrA & Conserved hypothetical protein & 0.02 \\
\hline 02975 & & PTS system, fructose-specific IIABC component, putative & 0.44 \\
\hline \multicolumn{4}{|l|}{ Upregulated } \\
\hline 01127 & & Conserved hypothetical protein & 2.08 \\
\hline 01366 & $\operatorname{trp} E$ & Anthranilate synthase component I & 6.32 \\
\hline 01367 & $\operatorname{trp} G$ & Anthranilate synthase component II & 5.77 \\
\hline 01368 & $\operatorname{trpD}$ & Anthranilate phosphoribosyltransferase & 6.80 \\
\hline 01369 & $\operatorname{trpC}$ & Indole-3-glycerol phosphate synthase & 6.60 \\
\hline 01370 & $\operatorname{trpF}$ & $N$-(5-Phosphoribosyl)anthranilate isomerase & 8.34 \\
\hline 01371 & $\operatorname{trp} B$ & Tryptophan synthase, beta subunit & 9.37 \\
\hline 01372 & $\operatorname{trp} A$ & Tryptophan synthase, alpha subunit & 9.09 \\
\hline 02449 & lacG & 6-Phospho- $\beta$-galactosidase & 2.04 \\
\hline 02451 & $\operatorname{lacF}$ & PTS system lactose-specific IIA component & 2.06 \\
\hline 02452 & $l a c D$ & Tagatose-1,6-diphosphate aldolase & 2.11 \\
\hline 02453 & lacC & Tagatose-6-phosphate kinase & 2.05 \\
\hline
\end{tabular}

the modification of lipopolysaccharides, outer-membrane proteins and membrane lipids, which in turn mediate resistance to different host defence elements that target the bacterial cell surface (Lamarche et al., 2008). Additionally, lytM, encoding a gly-gly endopeptidase (Ramadurai et al., 1999), and sceD, encoding a lytic transglycosylase (Stapleton et al., 2007), both involved in cell wall turnover, were upregulated twofold in the cwrA knockout.

Perhaps the most interesting transcriptional change observed was the upregulation of the dlt operon during exponential phase. The dlt operon is responsible for the D-alanylation of wall teichoic acids (WTAs) and lipoteichoic acids (LTAs), components of the cell envelope in $S$. aureus (Neuhaus et al., 1996). Teichoic acids represent the most abundant polyanions of the Gram-positive cell envelope (Fischer, 1994), and altering their charge properties through esterification with D-alanine has pleiotropic effects which can change autolytic enzyme activity (Wecke et al., 1996), cation binding (Hughes et al., 1973), susceptibility to cationic peptides (Peschel et al., 1999), virulence (Collins et al., 2002) and biofilm formation (Gross et al., 2001). DAlanylation also promotes coaggregation (Clemans et al., 1999), which is consistent with the clumping effect observed during in vitro growth. It is interesting that upregulation of the dlt operon occurred without a significant change in expression of either the arlSR two-component system, which has been demonstrated to repress $d l t$ operon expression (Koprivnjak et al., 2006), or the graRS two-component system, which has been shown to activate expression of the dlt operon (Herbert et al., 2007). Furthermore, dlt operon upregulation was independent of any significant change in expression of the capsular polysaccharide cap operon, another component of the cell envelope.

\section{Electron microscopy imaging}

Given the clumping phenotype and upregulation of the $d l t$ operon in response to deletion of $c w r A$, it seemed plausible that there might be a difference in the morphology of the cell envelope between the wild-type and knockout strains. In order to investigate, TEM was employed to image the two $S$. aureus strains. TEM thin sections revealed no discernible difference between the SH1000 and SH1000 $\mathrm{cwrA}: \mathrm{erm}$ strains with regard to their cell wall (Fig. 2). Both strains had a cell wall visible as an amorphous matrix $\sim 40 \mathrm{~nm}$ thick lying on top of the plasma membrane, which was visible as an encircling band of high density. Furthermore, there appeared to be no difference in formation of septa during cell division between the strains.

Interestingly, the wild-type $\mathrm{SH} 1000$ strain did differ from the $\mathrm{SH} 1000 \Delta c w r A:$ : erm mutant in the presence of a white band of low density residing proximally to the intracellular side of the plasma membrane. This intracellular space is most likely an artefact of processing the sample, which included a dehydration step before embedding in resin. During this dehydration the cytoplasm most likely contracted away from the less flexible cell envelope, resulting in the formation of a low-density space. In the cwrA knockout, this space may not have formed because the cell wall may have been compromised, allowing it to contract along with the cytoplasm during processing.

\section{Autolysis assays}

To verify the hypothesis suggested by the TEM images that the cwrA knockout has compromised cell wall integrity, autolysis assays were performed on SH1000 and 

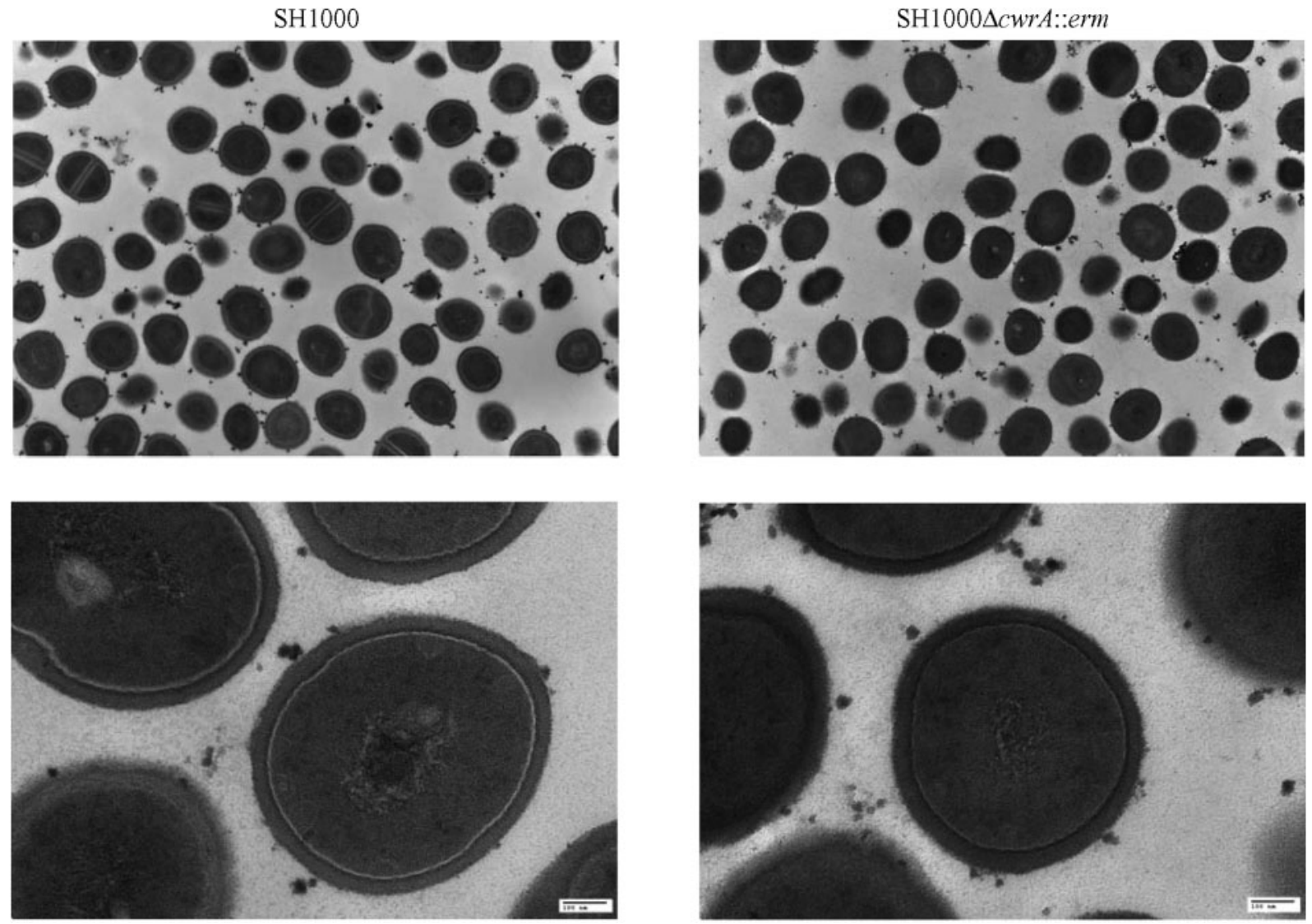

Fig. 2. TEM images of $\mathrm{SH} 1000$ and $\mathrm{SH} 1000 \Delta c w r A$ : : erm grown in MHB until stationary phase. Lower-panel images are a higher magnification of the upper-panel broad-field images; scale bars, $100 \mathrm{~nm}$.

SH1000 $\Delta$ cwrA:: erm. Fig. 3 demonstrates that the rate of autolysis for the knockout strain is indeed faster than that of the wild-type, supporting the role that cwrA plays in maintaining cell wall integrity. Several reports in the literature have demonstrated that lysis in response to inhibitory concentrations of cell wall-targeting antibiotics involves the activity of autolytic enzymes, as mutants defective in autolysin show reduced lysis in response to

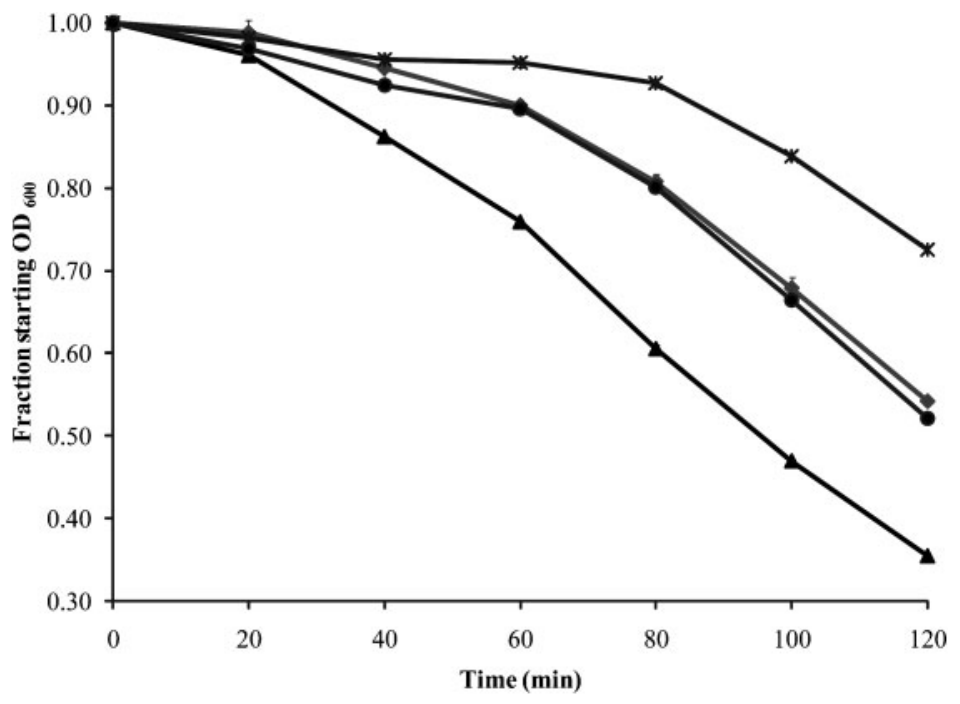

Fig. 3. Triton $\mathrm{X}-100$-induced autolysis. Autolysis was monitored for $\mathrm{SH} 1000$ and $\mathrm{SH} 1000 \Delta c w r A::$ erm grown in the absence (diamonds and triangles, respectively) or presence (asterisks and circles, respectively) of $350 \mathrm{ng}$ phosphomycin $\mathrm{ml}^{-1}$. Error bars, SD $(n=3)$. 

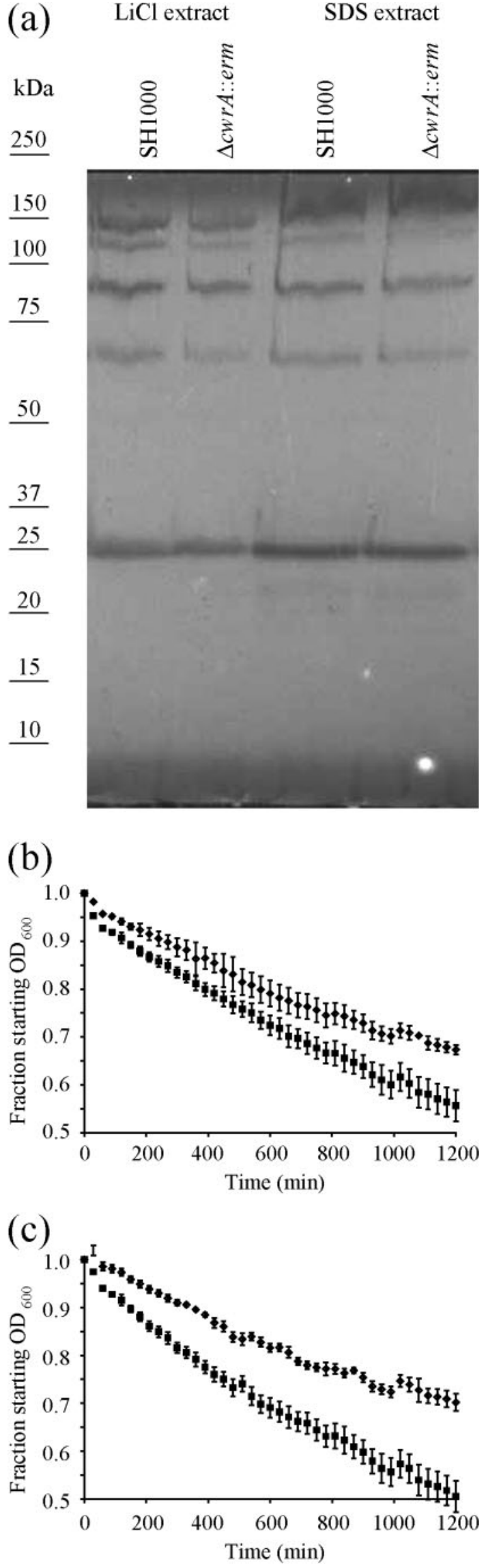

Fig. 4. In vitro autolysis assays. (a) Zymographic analysis of autolysins of $\mathrm{SH} 1000$ and $\mathrm{SH} 1000 \Delta c w r A$ : :erm extracted by either $\mathrm{LiCl}$ or SDS. The molecular mass ladder is Bio-Rad Precision Plus Protein Dual Color Standards. (b) In vitro autolysis using a freeze-thaw autolytic extract of $\mathrm{SH} 1000$ on cell wall extracted from $\mathrm{SH} 1000$ ( ) or $\mathrm{SH} 1000 \Delta c w r A:$ : erm (ם). (c) In vitro autolysis using a freeze-thaw autolytic extract of $\mathrm{SH} 1000 \Delta c w r A:$ :erm on cell wall extracted from $\mathrm{SH} 1000(\boldsymbol{\nabla})$ or $\mathrm{SH} 1000 \Delta c w r A$ : : erm $(\boldsymbol{\square})$. (b, c) Error bars, SD $(n=3)$.

antibiotic treatment (Oshida \& Tomasz, 1992; Tomasz et al., 1988). However, treatment with subinhibitory concentrations of $\beta$-lactams, which yields the production of hypocross-linked peptidoglycan that is more susceptible to in vitro enzymic degradation by autolysins (Qoronfleh \& Wilkinson, 1986), actually decreases the rate of autolysis in S. aureus. This autolytic protective effect induced by cell wall-targeting antibiotics has been shown to result from a decrease in transcription and/or the activity of several autolytic determinants, including $p b p B$, atl, sle1, SA0620 and lytM (Antignac et al., 2007). Since $c w r A$ is induced by cell wall-targeting antibiotics and its deletion resulted in a faster rate of autolysis, we investigated whether $c w r A$ was involved in this autolytic suppression. The autolysis assay was repeated in the presence of subinhibitory concentrations of phosphomycin (350 $\mathrm{ng} \mathrm{ml}^{-1}$ ). Once again, the cwrA knockout had a faster rate of autolysis than the wild-type (Fig. 3). However, the partial cell wall inhibition protection still resulted in a slower rate of autolysis for both SH1000 and SH1000 $\Delta c w r A$ ::erm. This result indicates that despite the fact that $c w r A$ is induced by cell wall-targeting antibiotics, its role is independent of the autolytic suppression induced by cell wall synthesis inhibition.

In order to confirm that increased autolysis was the result of decreased cell wall integrity and not simply increased autolysin production, as suggested by the transcriptional profiling data, peptidoglycan hydrolase profiles and in vitro autolysis assays were performed. As can be seen in Fig. 4(a), zymographic profiles of either $\mathrm{LiCl}$ or SDS autolytic extracts from SH1000 and SH1000 $\Delta c w r A$ : : erm were nearly identical, indicating that there is not an altered protein expression ratio for the major autolysins. However, when comparing the susceptibility of cell walls isolated from

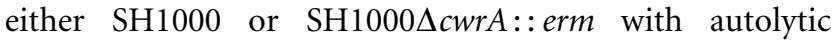
extracts from either strain, it became evident that cell walls isolated from SH1000 $\Delta c w r A::$ erm were more susceptible than cell walls isolated from SH1000 to degradation by autolytic extracts from either strain (Fig. 4b, c). This indicates that deletion of cwrA does indeed lead to a loss of cell wall integrity.

\section{Antibiotic and lysostaphin susceptibility}

The robust induction in response to cell wall-targeting antibiotics and the role in maintaining cell wall integrity suggest that $c w r A$ is involved in protecting $S$. aureus against antibacterial agents. As such, it would suggest that loss of 
cwrA would make S. aureus more susceptible to cell walltargeting antibiotics. Table 4 shows MIC values for several cell wall-targeting antibiotics against SH1000 and SH1000 $\Delta c w r A:: e r m$. For most compounds there was no change in MIC between the two strains, and for the few where SH1000 $\Delta c w r A$ : : erm was more susceptible (ticarcillin, amoxicillin and phosphomycin), the change was only twofold. Interestingly, although the $c w r A$ deletion strain showed an upregulation in the dlt operon, which purportedly makes the cells less susceptible to cationic peptides, there was no difference in MIC for polymyxin B, nisin and lysostaphin between the two strains. However, when a time course of lysis in response to lysostaphin was measured, there was a slight but significant increase in the rate of lysis for the $c w r A$ knockout strain (Supplementary Fig. S1).

\section{In vivo virulence}

With little effect in vitro, we wanted to investigate whether deletion of cwrA had more of a consequence in vivo. The $S$. aureus cell wall is a major component of how the host immune system is able to detect and respond to infection. Although cell wall-targeting antibiotics may be capable of inducing $c w r A$ in vitro, perhaps its major function lies in responding to host immune defences. A sepsis model was performed by infecting $\mathrm{CD}-1$ mice with wild-type and cwrA-deleted strains to determine virulence. At lower inocula, neither SH1000 nor SH1000 $\mathrm{cwrA}: \mathrm{erm}$ was virulent, and both showed a low degree of lethality. However, at the highest inoculum investigated, wild-type $S$. aureus showed $100 \%$ lethality, whereas the $c w r A$ knockout mutant showed a reduction of virulence. Fig. 5 shows a Kaplan-Meier survival curve for the highest inoculum, demonstrating that lethality with SH1000 occurred quickly and completely within 1 day, whereas even after 5 days most of the mice infected with SH1000 $\Delta$ cwrA::erm

Table 4. MIC $\left(\mu \mathrm{g} \mathrm{ml}^{-1}\right)$ of cell wall-targeting antibiotics and cationic peptides

\begin{tabular}{|lcc|}
\hline \multirow{2}{*}{$\begin{array}{l}\text { Antibiotic or } \\
\text { peptide }\end{array}$} & \multicolumn{2}{c|}{ Strain } \\
\cline { 2 - 3 } & SH1000 & SH1000 $\Delta$ cwrA : : erm \\
\hline Ticarcillin & 4 & 2 \\
Vancomycin & 1 & 1 \\
Amoxicillin & 0.5 & 0.25 \\
Penicillin & 1 & 1 \\
Ampicillin & 0.25 & 0.25 \\
Ceftazidime & 16 & 16 \\
Bacitracin & 16 & 16 \\
Phosphomycin & 2 & 1 \\
Polymyxin B & 512 & 512 \\
Nisin & 16 & 16 \\
Lysostaphin & 0.1 & 0.1 \\
\hline
\end{tabular}

survived. This indicates that $c w r A$ is important for virulence in an in vivo sepsis model of infection.

\section{DISCUSSION}

The staphylococcal cell wall is a dynamic structure that is paramount to the success of the organism. It withstands incredible turgor pressure, is intimately involved in cell division, both senses and interacts with the external environment, and is a major player in virulence (Beveridge, 1981). Since the discovery of penicillin, the bacterial cell wall has served as an effective target for antibacterial agents. Understanding the molecular and cellular response that occurs subsequent to peptidoglycan biosynthesis inhibition is of critical importance in understanding how pathogens adapt to, resist and evade killing by what are typically bactericidal agents. Herein, we studied $c w r A$, a gene that has appeared as the highest-fold upregulated in a variety of transcriptomic studies examining cell wall inhibition (Balibar et al., 2009; McAleese et al., 2006; Sobral et al., 2007).

Using a variety of transcriptional and translation fusion constructs and utilizing the bacterial luxABCDE operon for a luminescence readout, $c w r A$ was shown to be not only transcribed but also translated in response to treatment with cell wall-targeting antibiotics. Despite its massive transcriptional response, deletion of cwrA had little observable phenotype in vitro. Ultimately, transcriptional profiling, electron microscopy, and in vivo and in vitro autolysis assays demonstrated that deletion of cwrA resulted in a compromised cell wall with altered integrity. The role that cwrA plays in maintaining cell wall integrity could be structural or it could stem from a direct modulation of autolytic enzymes. Although in vitro growth

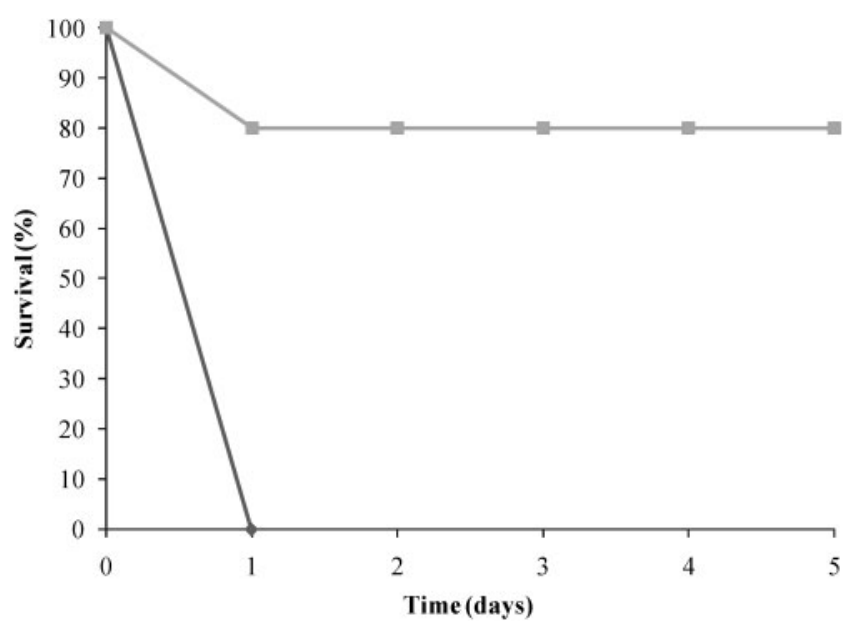

Fig. 5. Kaplan-Meier survival curve of CD-1 mice infected intraperitoneally with $\mathrm{SH} 1000$ (diamonds, dark grey) or $\mathrm{SH} 1000 \Delta c w r A$ : : erm (squares, light grey) at $4.2 \times 10^{8}$ c.f.u. in a sepsis model of infection $(n=5)$. 
was not significantly altered by cwrA deletion, the virulence of $S$. aureus was significantly altered in vivo. Using a 5 day mouse sepsis model of infection, deletion of $c w r A$ made $S$. aureus less virulent, a result that could be attributed to the loss of cell wall integrity in the knockout.

Perhaps most intriguing is that by fusing the entire gene or the putative promoter of $c w r A$ to a luciferase gene operon, we successfully established $S$. aureus strains that are able to produce robust bioluminescence signals as a specific response to chemicals that block cell wall biosynthesis. Often, high-throughput screens performed during antimicrobial discovery efforts result in the identification of compounds with unknown mechanisms of action. The strains constructed here should be useful for the characterization of such compounds or could be used directly to identify antimicrobial agents with specific cell walldirected mechanisms. These strains are responsive to a broad range of cell wall inhibitors that interfere with numerous steps in peptidoglycan biosynthesis.

Most of the cell wall inhibitors tested here triggered the luminescence signal within $2 \mathrm{~h}$ of the treatment. However, it is interesting that hymeglusin required more than $4 \mathrm{~h}$ in order to induce the expression of the reporter luciferase to a detectable level. Such a delay in response is correlated with the fact that this compound inhibits an early stage of UPP synthesis. It is also possible that the regulation that induces the cell wall stimulon is caused by the accumulation of peptidoglycan precursors, which is on a biosynthetic branch required for the generation of cell walls that is different from that inhibited by hymeglusin. Our observation that phosphomycin induces the strongest signal among all the compounds tested would be consistent with such a hypothesis. It is also intriguing that vancomycin caused only a transient signal that lasted less than $1 \mathrm{~h}$.

Since these strains were constructed, similar $S$. aureus cell wall reporter strains have been described in which several promoters from other cell wall stimulon members are fused to a lacZ gene (Steidl et al., 2008). However, the strains described here do not require the addition of an exogenous substrate, offer much lower background, and produce a more robust readout that is based on a luminescence signal.

\section{ACKNOWLEDGEMENTS}

We thank Simon J. Foster (University of Sheffield, UK) for providing strain SH1000, Chia Y. Lee (University of Kansas Medical Center, Kansas City, KS) for providing us with plasmid pCL84 and strain CYL316, Nicki Watson (Whitehead Institute, Cambridge, MA) for TEM sample preparation and imaging, and Steven J. Projan and Neil S. Ryder for a careful reading of the manuscript.

\section{REFERENCES}

Amako, K., Umeda, A. \& Murata, K. (1982). Arrangement of peptidoglycan in the cell wall of Staphylococcus spp. J Bacteriol 150, 844-850.
Antignac, A., Sieradzki, K. \& Tomasz, A. (2007). Perturbation of cell wall synthesis suppresses autolysis in Staphylococcus aureus: evidence for coregulation of cell wall synthetic and hydrolytic enzymes. J Bacteriol 189, 7573-7580.

Archer, G. L. (1998). Staphylococcus aureus: a well-armed pathogen. Clin Infect Dis 26, 1179-1181.

Balibar, C. J., Shen, X. \& Tao, J. (2009). The mevalonate pathway of Staphylococcus aureus. J Bacteriol 191, 851-861.

Bernsel, A., Viklund, H., Hennerdal, A. \& Elofsson, A. (2009). TOPCONS: consensus prediction of membrane protein topology. Nucleic Acids Res 37, W465-W468.

Beveridge, T. J. (1981). Ultrastructure, chemistry, and function of the bacterial wall. Int Rev Cytol 72, 229-317.

Clemans, D. L., Kolenbrander, P. E., Debabov, D. V., Zhang, Q., Lunsford, R. D., Sakone, H., Whittaker, C. J., Heaton, M. P. \& Neuhaus, F. C. (1999). Insertional inactivation of genes responsible for the D-alanylation of lipoteichoic acid in Streptococcus gordonii DL1 (Challis) affects intrageneric coaggregations. Infect Immun 67, 24642474.

Collins, L. V., Kristian, S. A., Weidenmaier, C., Faigle, M., Van Kessel, K. P., Van Strijp, J. A., Gotz, F., Neumeister, B. \& Peschel, A. (2002). Staphylococcus aureus strains lacking D-alanine modifications of teichoic acids are highly susceptible to human neutrophil killing and are virulence attenuated in mice. J Infect Dis 186, 214-219.

Fischer, W. (1994). Lipoteichoic acid and lipids in the membrane of Staphylococcus aureus. Med Microbiol Immunol 183, 61-76.

Giesbrecht, P., Kersten, T., Maidhof, H. \& Wecke, J. (1998). Staphylococcal cell wall: morphogenesis and fatal variations in the presence of penicillin. Microbiol Mol Biol Rev 62, 1371-1414.

Gross, M., Cramton, S. E., Gotz, F. \& Peschel, A. (2001). Key role of teichoic acid net charge in Staphylococcus aureus colonization of artificial surfaces. Infect Immun 69, 3423-3426.

Guzman, L. M., Belin, D., Carson, M. J. \& Beckwith, J. (1995). Tight regulation, modulation, and high-level expression by vectors containing the arabinose $\mathrm{P}_{\mathrm{BAD}}$ promoter. J Bacteriol 177, 4121-4130.

Herbert, S., Bera, A., Nerz, C., Kraus, D., Peschel, A., Goerke, C., Meehl, M., Cheung, A. \& Gotz, F. (2007). Molecular basis of resistance to muramidase and cationic antimicrobial peptide activity of lysozyme in staphylococci. PLoS Pathog 3, e102.

Horsburgh, M. J., Aish, J. L., White, I. J., Shaw, L., Lithgow, J. K. \& Foster, S. J. (2002). $\sigma^{\mathrm{B}}$ modulates virulence determinant expression and stress resistance: characterization of a functional $r s b U$ strain derived from Staphylococcus aureus 8325-4. J Bacteriol 184, 54575467.

Hughes, A. H., Hancock, I. C. \& Baddiley, J. (1973). The function of teichoic acids in cation control in bacterial membranes. Biochem $J$ 132, 83-93.

Kadurugamuwa, J. L., Sin, L., Albert, E., Yu, J., Francis, K., DeBoer, M., Rubin, M., Bellinger-Kawahara, C., Parr, T. R., Jr \& Contag, P. R. (2003). Direct continuous method for monitoring biofilm infection in a mouse model. Infect Immun 71, 882-890.

Klevens, R. M., Morrison, M. A., Fridkin, S. K., Reingold, A., Petit, S., Gershman, K., Ray, S., Harrison, L. H., Lynfield, R. \& other authors (2006). Community-associated methicillin-resistant Staphylococcus aureus and healthcare risk factors. Emerg Infect Dis 12, 19911993.

Koprivnjak, T., Mlakar, V., Swanson, L., Fournier, B., Peschel, A. \& Weiss, J. P. (2006). Cation-induced transcriptional regulation of the dlt operon of Staphylococcus aureus. J Bacteriol 188, 3622-3630.

Kuroda, M., Kuroda, H., Oshima, T., Takeuchi, F., Mori, H. \& Hiramatsu, K. (2003). Two-component system VraSR positively 
modulates the regulation of cell-wall biosynthesis pathway in Staphylococcus aureus. Mol Microbiol 49, 807-821.

Lamarche, M. G., Wanner, B. L., Crepin, S. \& Harel, J. (2008). The phosphate regulon and bacterial virulence: a regulatory network connecting phosphate homeostasis and pathogenesis. FEMS Microbiol Rev 32, 461-473.

Lee, C. Y., Buranen, S. L. \& Ye, Z. H. (1991). Construction of singlecopy integration vectors for Staphylococcus aureus. Gene 103, 101-105.

McAleese, F., Wu, S. W., Sieradzki, K., Dunman, P., Murphy, E., Projan, S. \& Tomasz, A. (2006). Overexpression of genes of the cell wall stimulon in clinical isolates of Staphylococcus aureus exhibiting vancomycin-intermediate- $S$. aureus-type resistance to vancomycin. J Bacteriol 188, 1120-1133.

McCallum, N., Spehar, G., Bischoff, M. \& Berger-Bachi, B. (2006). Strain dependence of the cell wall-damage induced stimulon in Staphylococcus aureus. Biochim Biophys Acta 1760, 1475-1481.

Muthaiyan, A., Silverman, J. A., Jayaswal, R. K. \& Wilkinson, B. J. (2008). Transcriptional profiling reveals that daptomycin induces the Staphylococcus aureus cell wall stress stimulon and genes responsive to membrane depolarization. Antimicrob Agents Chemother 52, 980-990.

Navarre, W. W. \& Schneewind, O. (1999). Surface proteins of Grampositive bacteria and mechanisms of their targeting to the cell wall envelope. Microbiol Mol Biol Rev 63, 174-229.

Neuhaus, F. C., Heaton, M. P., Debabov, D. V. \& Zhang, O. (1996). The dlt operon in the biosynthesis of D-alanyl-lipoteichoic acid in Lactobacillus casei. Microb Drug Resist 2, 77-84.

Oshida, T. \& Tomasz, A. (1992). Isolation and characterization of a Tn551-autolysis mutant of Staphylococcus aureus. J Bacteriol 174, 4952-4959.

Peschel, A., Otto, M., Jack, R. W., Kalbacher, H., Jung, G. \& Gotz, F. (1999). Inactivation of the dlt operon in Staphylococcus aureus confers sensitivity to defensins, protegrins, and other antimicrobial peptides. J Biol Chem 274, 8405-8410.

Qoronfleh, M. W. \& Wilkinson, B. J. (1986). Effects of growth of methicillin-resistant and -susceptible Staphylococcus aureus in the presence of beta-lactams on peptidoglycan structure and susceptibility to lytic enzymes. Antimicrob Agents Chemother 29, 250-257.
Ramadurai, L., Lockwood, K. J., Nadakavukaren, M. J. \& Jayaswal, R. K. (1999). Characterization of a chromosomally encoded glycylglycine endopeptidase of Staphylococcus aureus. Microbiology 145, 801808.

Sobral, R. G., Jones, A. E., Des Etages, S. G., Dougherty, T. J., Peitzsch, R. M., Gaasterland, T., Ludovice, A. M., de Lencastre, H. \& Tomasz, A. (2007). Extensive and genome-wide changes in the transcription profile of Staphylococcus aureus induced by modulating the transcription of the cell wall synthesis gene murF. J Bacteriol 189, 2376-2391.

Stapleton, M. R., Horsburgh, M. J., Hayhurst, E. J., Wright, L., Jonsson, I. M., Tarkowski, A., Kokai-Kun, J. F., Mond, J. J. \& Foster, S. J. (2007). Characterization of IsaA and SceD, two putative lytic transglycosylases of Staphylococcus aureus. J Bacteriol 189, 7316-7325.

Steidl, R., Pearson, S., Stephenson, R. E., Ledala, N., Sitthisak, S., Wilkinson, B. J. \& Jayaswal, R. K. (2008). Staphylococcus aureus cell wall stress stimulon gene-lac $Z$ fusion strains: potential for use in screening for cell wall-active antimicrobials. Antimicrob Agents Chemother 52, 2923-2925.

Tomasz, A., Moreillon, P. \& Pozzi, G. (1988). Insertional inactivation of the major autolysin gene of Streptococcus pneumoniae. J Bacteriol 170, 5931-5934.

Utaida, S., Dunman, P. M., Macapagal, D., Murphy, E., Projan, S. J., Singh, V. K., Jayaswal, R. K. \& Wilkinson, B. J. (2003). Genome-wide transcriptional profiling of the response of Staphylococcus aureus to cell-wall-active antibiotics reveals a cell-wall-stress stimulon. Microbiology 149, 2719-2732.

Wecke, J., Perego, M. \& Fischer, W. (1996). D-Alanine deprivation of Bacillus subtilis teichoic acids is without effect on cell growth and morphology but affects the autolytic activity. Microb Drug Resist 2, 123-129.

Wilding, E. l., Brown, J. R., Bryant, A. P., Chalker, A. F., Holmes, D. J., Ingraham, K. A., lordanescu, S., So, C. Y., Rosenberg, M. \& Gwynn, M. N. (2000). Identification, evolution, and essentiality of the mevalonate pathway for isopentenyl diphosphate biosynthesis in Gram-positive cocci. J Bacteriol 182, 4319-4327.

Edited by: J. H. Cove 